\section{Classic fail}

W e are notoriously bad at probabilistic thinking. For one thing, extreme events loom inordinately large in our minds. More people fear flying than driving even though the risks of dying in the latter activity are about 100 times greater, because an aircraft accident feels so much more catastrophic and awful.

But sometimes it really is the rare events that matter most. This is the case in fracture of brittle materials, which is typically initiated at defects such as scratches or cracks. The largest defects matter most for failure, but they are also likely to be much more rare than small defects. The longer a fibre, say, the more likely it is to contain a large defect if these appear at random - and so the force needed to break the fibre should decrease as the fibre gets longer.

This kind of fracture is one of the best-studied problems in materials science, dating at least back to early work on fibre-reinforced materials and the realization that fine fibres of brittle substances such as glass can be very strong because their small surface area means there is little chance of it including a strength-compromising flaw. The problem was studied by A. A. Griffith in the 1920s, and an empirical solution for fracture strengths in terms of defect probabilities was given by Waloddi Weibull in $1951^{1}$. But Weibull's model, although widely used, has never been given a firm theoretical justification.

In particular, what is the exact relationship between the length of a fibre and the force needed to fracture it? Fontana and Palffy-Muhoray answer that question from first principles by making a connection to a quirk of probability theory that has been known since its earliest beginnings in the eighteenth century, called the St Petersburg paradox ${ }^{2}$.

This was described in a private letter of 1713 by the Swiss mathematician Nicolaus Bernoulli. He mentioned it to his even more illustrious brother Daniel, most famous now for his work on fluid flow, who published a paper on the subject in the journal of the Imperial Academy of Sciences of St Petersburg in 1738 . To the Bernoullis it was a problem of risk, expressed as a game. The modern version typically runs as follows. A banker offers a person the chance to play, for a fee, a coin-flipping game with payoffs. The rule is that the player will be paid when the coin lands heads up, as it surely will at some point. When it occurs on the $n$th flip, the prize is $2^{n}$ dollars. What entry fee should the player be willing to pay?

That can be deduced from the expectation value of the payout for a single game, which is found by adding up each outcome multiplied by its probability of occurrence: the sum of $1 / 2^{n} \times 2^{n}$ for all $n-$ which is infinity, even though the typical payout will be very small. The paradox is created by the mere possibility of rare, extreme outcomes, and has stimulated much discussion among economists about how rational agents should make decisions based on 'utility', or the theoretically anticipated benefits.

Fontana and Palffy-Muhoray show that this paradox maps onto the fibre failure problem, where longer fibres have a greater chance of harbouring

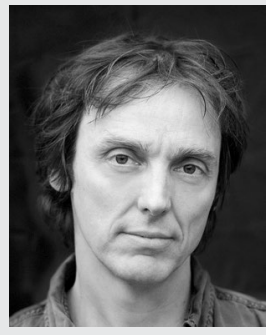

Philip Ball

rare large defects. Just as it has been shown that the largest expected cluster of tails in a series of $N$ coin tosses depends on the logarithm of $N$, so the size of the largest defect in a fibre of length $L$ varies as $\ln (L)$. If then the fracture force for the fibre is negatively proportional to this largest-defect size, the fracture force varies as $-\ln (L)$.

The researchers test this prediction for polyester and polyamide fibres, using a motor and pulley system, over a remarkable range of lengths: six orders of magnitude, from 1 $\mathrm{mm}$ to $1 \mathrm{~km}$. Only over such a range should the small differences between the log-linear St Petersburg relationship and the nonlinear Weibull prediction become apparent. And they do: the former relationship fits the results best, confirming that the peculiar game that puzzled the Bernoullis seems to have a real-world manifestation with broad and important practical ramifications.

Published online: 23 July 2020 https://doi.org/10.1038/s41563-020-0752-2

References

1. Weibull, W. J. Appl. Mech. 18, 293-297 (1951).

2. Fontana, J. \& Palffy-Muhoray, P. Phys. Rev. Lett. 124, 245501 (2020). 